TRABAJOS ORIGINALES

\title{
Notas sobre Chloraea undulata "Orquídea de Lima" y su registro en las lomas de Asia, Cañete, Perú
}

\section{Notes on the "Lima orchid" Chloraea undulata and its record in the hills of Asia, Cañete, Peru}

\section{Miguel Lleellish Juscamayta}

Servicio Nacional Forestal y de Fauna Silvestre SERFOR. Ministerio de Agricultura y Riego. Calle 17 N 355 Urb. El Palomar, San Isidro, Lima - Perú. [mlleellish@gmail.com]

Email: mlleellish@serfor.gob.pe

\section{Resumen}

Chloraea undulata es una orquídea endémica del Perú. Originalmente colectada por Antonio Raimondi en el siglo XIX, fué reconocida como una especie característica de las lomas de Lima hasta mediados del siglo pasado. Desde entonces, la especie ha sido considerada desaparecida de las lomas costeras y especialmente del Cerro Amancaes, su localidad tipo, que hoy integra el casco urbano de la capital. En el presente trabajo, se documenta su presencia en las lomas de Asia $100 \mathrm{~km}$ al sur de Lima y se valora la importancia de este hallazgo para la conservación de la especie y su hábitat.

Palabras clave: Chloraea undulata; lomas costeras; desierto peruano; ecosistemas frágiles.

\section{Abstract}

Chloraea undulata is an orchid endemic to Peru. Originally collected by Antonio Raimondi in XIX century, until first half of the last century it has been regarded as a species characteristic of the hills around Lima. The "Lima orchid" Chloraea undulata is considered to be in decline in the hills of Lima province, especially in the type localities of Cerro Amancaes and Cerro El Agustino where urban growth and expansion has been significant. In this paper its presence in the hills of Asia District, $100 \mathrm{~km}$ south of Lima, is documented, and we also analyzed the implications of this finding for the species conservation and their habitat.

Keywords: Chloraea undulata; coastal hills; Peruvian desert; fragile ecosystem.

Citación:

Lleellish Juscamayta M. 2015. Notas sobre Chloraea undulata "Orquídea de Lima" y su registro en las lomas de Asia, Cañete, Perú. Revista peruana de biología 22(3): 309 - 314 (Diciembre 2015). doi: http://dx.doi. org/10.15381/rpb.v22i3.11436

$\begin{array}{ll}\text { Presentado: } & 15 / 12 / 2014 \\ \text { Aceptado: } & 06 / 12 / 2015\end{array}$

Aceptado: $\quad 06 / 12 / 2015$

Publicado online: 18/12/2015
Información sobre los autores:

El autor manifoesta que no incurre en conflictos de intereses.

Permiso de colecta:

El estudio se realizó bajo la autorización RD Nº 294 - 2014-MINAGRIDGFFS/DGEFFS- emitida por la Dirección General Forestal y de Fauna Silvestre del Ministerio de Agricultura- actualmente Servicio Nacional Forestal y Fauna Silvestre (SERFOR). 


\section{Introducción}

Las lomas costeras son ecosistemas notables localizados en el desierto costero que comparten Perú y Chile (Lleellish et al. 2015). Alimentadas por la humedad de las neblinas procedentes del Océano Pacifíco, las lomas han permitido el asentamiento de especies vegetales perfectamente adaptadas al medio, algunas de ellas emblemáticas, como el "amancaes" Ismene amancaes (Ker Gawl.) Herb., y Chloraea undulata Raimondi ex Colunga, la "orquídea de Lima”, una de las 4 especies de orquídeas reportadas para estos ecosistemas de neblina (Trujillo \& Delgado 2011).

Las lomas de Asia se ubican a $100 \mathrm{~km}$ al sur de la ciudad de Lima en el distrito de Asia, provincia de Cañete, departamento de Lima, en territorio de la Comunidad Campesina de Asia (Fig. 1). El área está formada por un conjunto de colinas, quebradas y cumbres de cerros entre los 200 y 1200 de elevación, que se cubren de una vegetación herbácea estacional durante los meses de primavera e invierno.

Chloraea undulata (Fig. 2), orquídea endémica del Perú fue descrita por Raimondi (in Colunga 1878) a partir del material colectado por el sabio en las lomas costeras cercanas a la ciudad de Lima (registro 739 USM, Fig. 3), como las del Cerro Amancaes y Cerro San Jerónimo en el Rímac, reconocidas como localidades tipo de la especie.

\section{Chloraea en el Perú y en las lomas costeras}

Chloraea es un género no-monofilético endémico de Sudamérica (Cisternas et al. 2012), que se distribuye de manera disjunta en tres áreas geográficas muy distantes entre sí: el área I

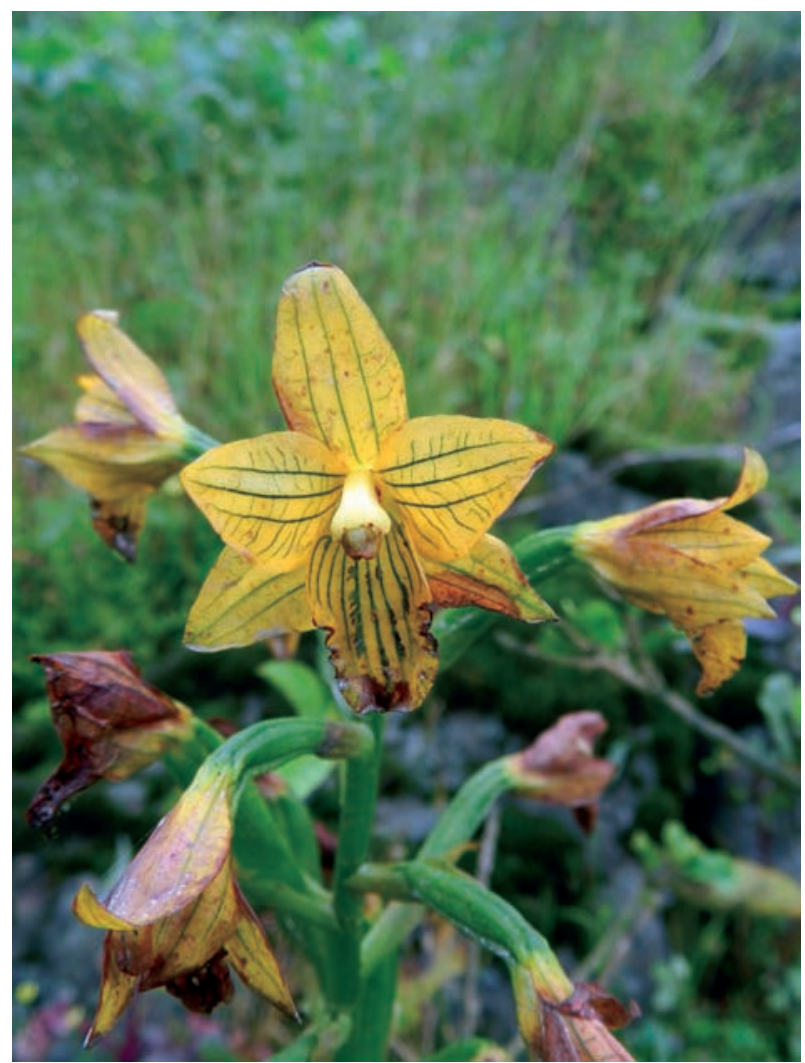

Figura 2. Ejemplar en floración de Chloraea undulata en las lomas de Asia, mostrando los pétalos de color amarillo y la venación verdosa.

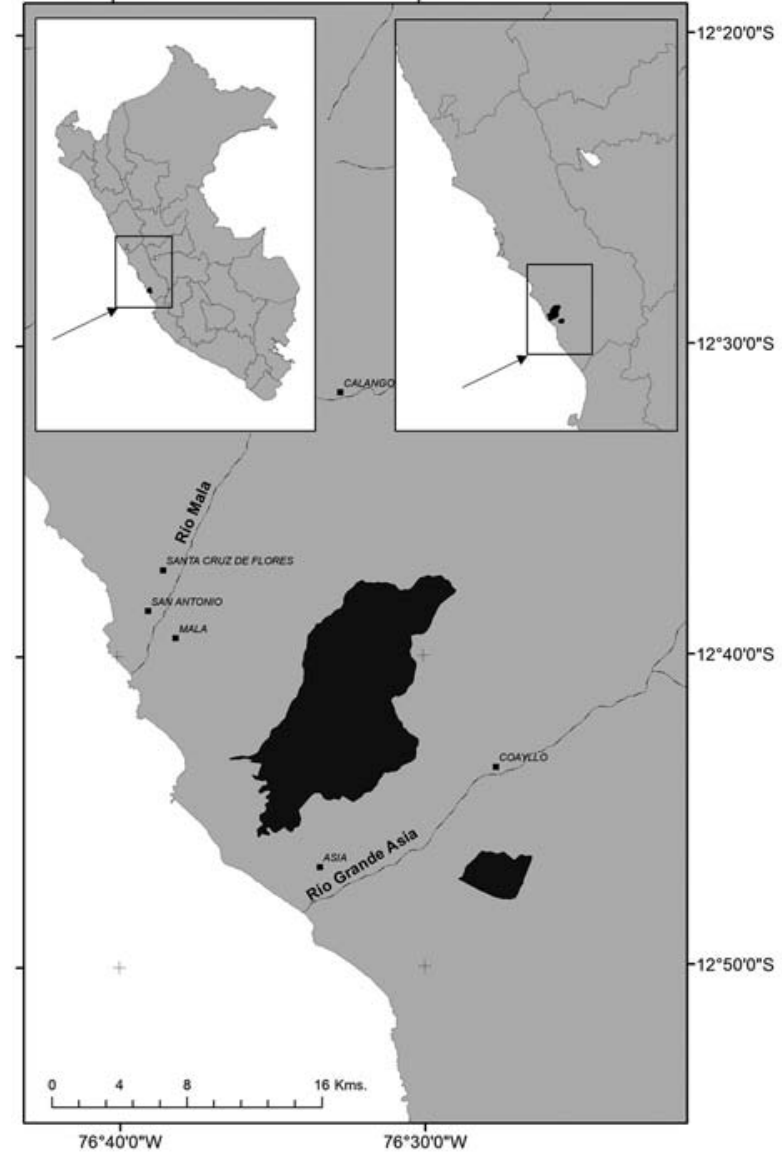

Figura 1. Mapa de ubicación de las Lomas de Asia en el Departamento de Lima.

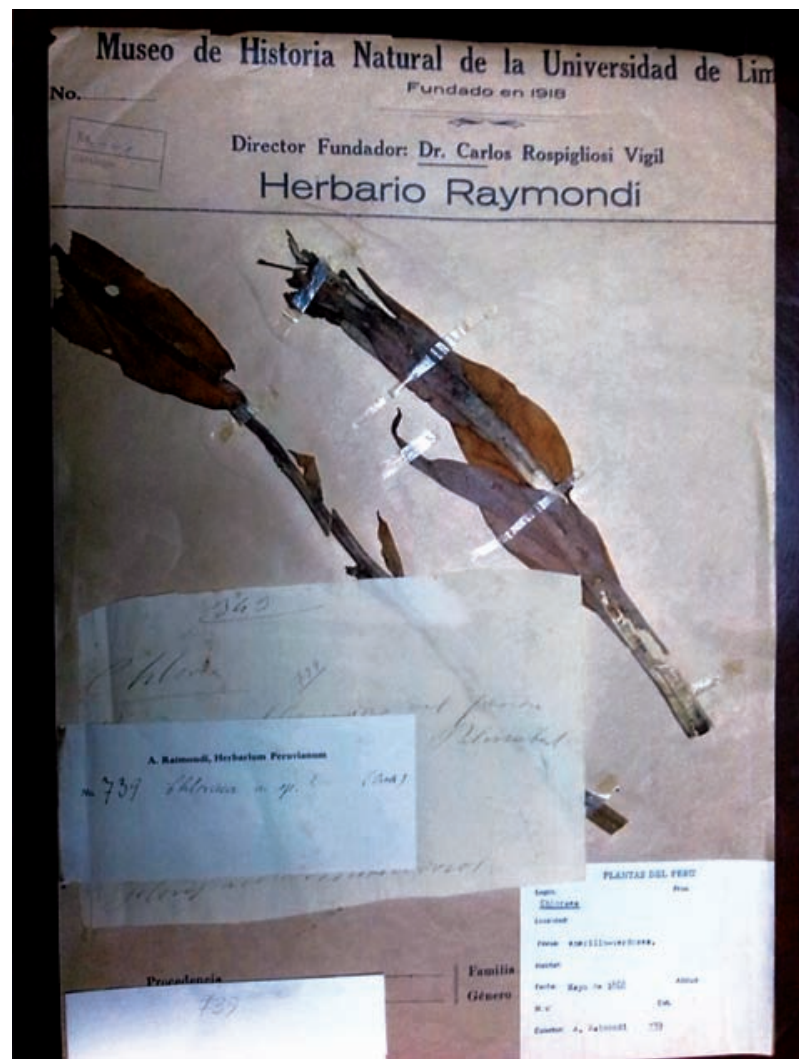

Figura 3. Ejemplar representativo de Chloraea undulata colectado por Raimondi en 1868. (A. Raimondi 739, USM). 


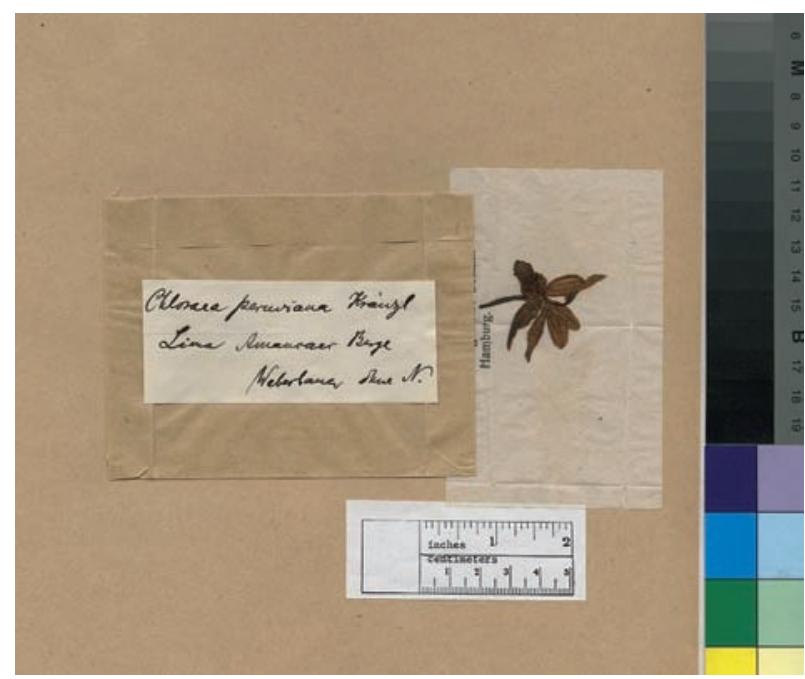

Figura 4. Holotipo de Chloraea peruviana Kraenzl. (Sinónimo de Chloraea undulata) colectado por Weberbauer en el cerro Amancaes (A. Weberbauer HBG501465).

Patagónica-Chilena, que corresponde a la mayor parte de Chile desde los $30^{\circ} \mathrm{S}$ hasta la Patagonia, área II Tucumano- Boliviano, que incluye las especies de Perú región sudeste de Bolivia, y el norte de Argentina; y área III Sudbrasilo-Platense localizada entre Brasil y Argentina (Hauman 1922).

Las especies peruanas de Chlorea comprenden a C. undulata, C. reticulata Schltr., C multilineolata C.Schweinf., C. densipapillosa C.Schweinf. (Scheweinfurth 1970) y C. septentrionalis M.N.Correa (Correa 1969), esta última reportada para los bosques montanos de La Libertad (Sagástegui et al. 1991).

De acuerdo con The Plant List (2015) C. undulata es un nombre válido y Chloraea peruviana Kraenzl. (Fig. 4) es considerada sinónima de C. undulata (Pupulin 2012, Garay \& Romero 1998, Schweinfurth 1970).

Chloraea undulata habita las lomas costeras y regiones interandinas (Brako \& Zarucchi 1993), en las cuencas de los rios Jequetepeque y Chicama, en los departamentos de La Libertad y Cajamarca, y en la cuenca del río Rímac en el departamento de Lima (Roque \& León 2006).

La revisión de los registros con que cuenta el Herbario de San Marcos, señalan como hábitats más frecuentes de la especie, en la actualidad; las laderas rocosas entre los 2600 y 2900 m de altitud, en las localidades de Arahuay, Lachanqui y Collo en la provincia de Canta, y el Bosque de Zarate en la provincia de Huarochiri, todas las localidades en el departamento de Lima. Dichas localidades están ubican a mayor altitud que las lomas costeras, ecosistemas cuya máxima altitud bordea los $1200 \mathrm{~m}$ de altitud, en los pisos más bajos de los flancos andinos.

Fuera de Lima, C. undulata ha sido registrada en Chincheros, departamento de Cusco (Franquemont 1990) y las cuencas de los ríos Jequetepeque y Chicama en el departamento de La Libertad, a aproximadamente 3100 m de altitud (Sagastegui \& Dillon 1991).

Trujillo (2013) mencionó que el último registro de la especie en las lomas costeras fue el de Ramón Ferreyra en el año 1952 en las lomas de Iguanil provincia de Huaral (aproximadamente

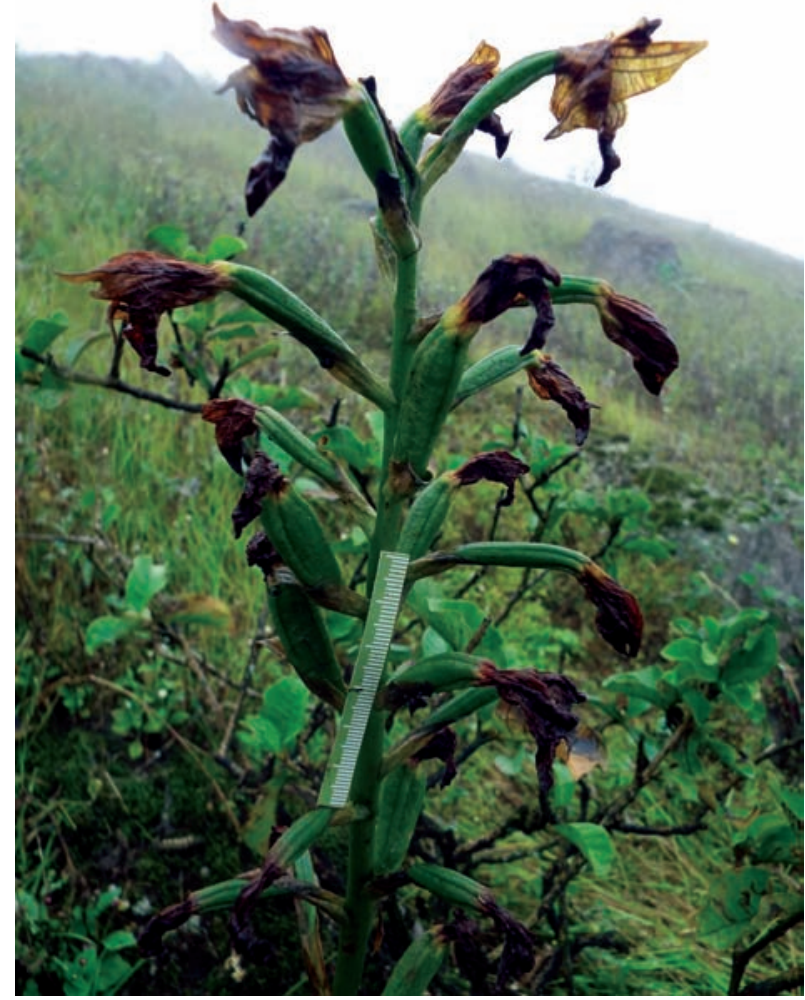

Figura 5. Escapo floral de Chloraea undulata portando 23 flores. Al fondo, se aprecia el hábitat característico conocido como "loma herbácea".

a $72 \mathrm{~km}$ al norte de Lima). Desde entonces $C$. undulata no ha sido observada en estas comunidades a pesar de los esfuerzos realizados para conocer su estado actual de conservación (Trinidad et al. 2012, Lleellish et al. 2015).

\section{Redescubriendo la Orquídea de Lima en las lomas costeras}

El registro de un único ejemplar de $C$. undulata tuvo lugar en setiembre de 2014 en la loma de Asia (Quebrada Gonzalillo, 79¹7’19”W; 1245'3”S), en el hábitat denominado loma herbácea, a una altitud de $490 \mathrm{~m}$ (Fig. 5).

La planta fue hallada en un desfiladero o camino divisorio entre dos laderas; protegida por varias rocas grandes que creaban en conjunto un microhábitat más húmedo que el área circundante, y a pocos metros de la base de una torre de alta tensión. Dicha ubicación dificultaba el pastoreo de ganado vacuno y el transito frecuente por los pobladores locales. Asimismo, el avistamiento de un "jergón de costa" Bothrops pictus (Tschudi 1945), especie amenazada, sumamente rara de encontrar, confirmaba lo poco visitada de la zona.

Chloraea undulata fue ubicada en territorio de la Comunidad Campesina de Asia, motivo por el cual, respetando el pedido de la población local sobre las precauciones con las especies valiosas o únicas, se evitó su colecta. No obstante, fue posible obtener datos morfométricos y biológicos en campo, durante los meses de setiembre y octubre de 2014, los que se detallan a continuación:

Altura de la planta $79 \mathrm{~cm}$ incluyendo el escapo floral. Tallo foliáceo cubierto por hojas envainantes. Hojas 8 , ovada- lan- 


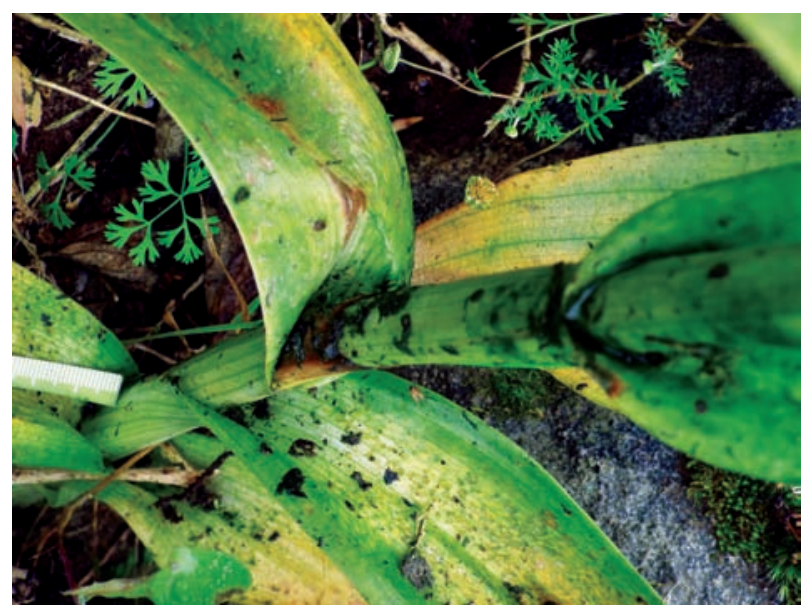

Figura 6. Chloraea undulata, detalle del tallo con la típica venación verdosa. Se muestran las hojas envainadoras e imbricadas en la base.

ceoladas (Fig.6). Hoja basal más grande $17 \mathrm{~cm}$ de largo por 5.5 $\mathrm{cm}$ de ancho; hoja distal más pequeńa $8 \mathrm{~cm}$ de largo por 2.5 $\mathrm{cm}$ de ancho. Longitud de la espiga floral $27 \mathrm{~cm}$. Número total de flores 23 (todas con floración exitosa). Cápsula o botón de semilla $3.2 \mathrm{~cm}$ a $4.2 \mathrm{~cm}$ de longitud y $1.0 \mathrm{~cm}$ a $1.2 \mathrm{~cm}$ de ancho. Color de la flor amarillo con venas verdes, notorias, reticuladas. Sépalos aproximadamente de $3 \mathrm{~cm}$ de longitud. Aunque no se observó ni se tomaron datos de la raíz, es importante mencionar los apuntes de Ramón Ferreyra sobre la coloración clara y "casi transparente" de los tubérculos de un ejemplar de las lomas de Chancay (ficha de registro 8748 USM, año 1952).

La floración fue observada entre setiembre y la primera semana de octubre. El inicio de la fructificación se observó en la última quincena de octubre. Durante este período, las hojas basales empezaron a secarse.

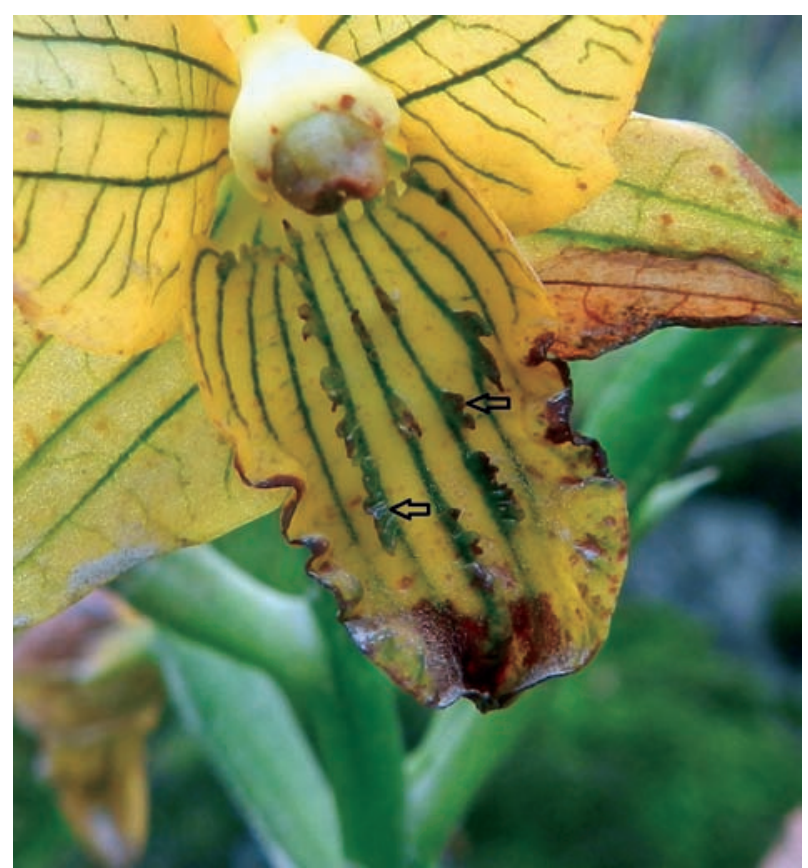

Figura 7. Detalle del labelo de Chloraea undulata mostrando los apéndices engrosados y algo falcados, siguiendo el curso de las venas.
Como elementos florísticos sobresalientes símpatricos con C. undulata se encontró a Croton alnifolius Lam., Solanum americanum Mill., Sonchus oleraceus L., Fuertesimalva limensis (L.) Fryxell, F. peruviana (L.) Fryxell, Crassula connata (Ruiz \& Pav.) A. Berger, Raymundochloa trachyanta, Fumaria capreolata L. y Oxalis megalorrhiza Jacq.

\section{Caracteres específicos de Chloraea undulata según Raimondi}

Miguel de Fernández Colunga (1878), médico y amigo de confianza de Raimondi, recogió en su libro, Lecciones de Botánica, los caracteres específicos dados por el sabio a la especie, los cuales constituyen su protólogo:

Chloraea undulata. Caule sesquipedalis, folioso, foliis ellipticooblongis, basi vaginantibus, racemo terminalis, multifloro (12-20:), perigonii phyllis e - ternis oblongis subaequalibus, apice reflexis. Perigonii phyllis lateralibus internis, ovalibus obtusis. Labello unguiculato obsolete obtuso, trilobo, lobis lateralibus obtusis, integris. Intermedio obtuso margine undulato, minute crenato, disco venis 5-7-9 appendicibus nunc falcatis, nunc lamellatis, nunc tridentatis.

Colunga (1878), se refiere a esta planta como "...tal vez la única especie indígena de Chloraea en el Perú” y asimismo, menciona que "... se halla en las inmediaciones de los cerros de Lima: tiene una talla de un metro poco más o menos: con hojas elíptico-oblongas: las flores dispuestas en racimos: con el periancio de un color amarillo dorado con estrías verdosas: el labellum unguiculado y trilobo" (p. 187)

El ejemplar encontrado en Asia posee las principales características de diagnóstico para $C$. undulata: racimo terminal con más de 20 flores amarillas con venas verdes, sépalos con más de $2.5 \mathrm{~cm}$ de longitud, labelo sub-simple o discretamente trilobado, el lóbulo intermedio obtuso con el margen ondulado (Fig. 7). El disco del labelo con unas cuantas papilas engrosadas y falcadas siguiendo la orientación de las venas, sin llegar a constituir un conjunto denso (como en el caso de $C$. densipapillosa: Schweinfurth 1970). Esta descripción concuerda también con los detalles plasmados en la acuarela de H. del Garnier (Raimondi et al. 2010, Fig. 8), uno de los ilustradores que colaboraron con Raimondi.

\section{Consecuencias para la gestión y conservación de la loma de Asia}

En el Perú C. undulata está considerada en peligro crítico de extinción (D.S. 043-2006-AG). Roque y León (2006) justificaron el grado de amenaza de la especie en base a una reducida extensión de la presencia (menor a $5000 \mathrm{~km}^{2}$ ), su ocurrencia en no más de 5 localidades y una disminución continúa en su área de extensión o calidad de hábitat. El hecho de que $C$. undulata no haya sido reportada durante más de 60 años apoya la evaluación realizada sobre la especie y su grado critico de amenaza.

Las lomas costeras son consideradas como ecosistemas frágiles según lo establece la Ley $\mathrm{N}^{\circ} 28611$, y alguna de ellas integran la lista nacional de ecosistemas frágiles ( $\left.R M N^{\circ} 0274-M I N A G R I\right)$. $\mathrm{Su}$ fragilidad se manifiesta en la poca resiliencia que tienen para afrontar las graves amenazas antrópicas que afectan su funcionamiento, como lo son: el avance del casco urbano, sobrepastoreo, contaminación por residuos sólidos, minería no metálica, tendido de líneas eléctricas y la ampliación de la frontera agrícola (Lleellish et al. 2015). Como producto de dichas amenazas, la 


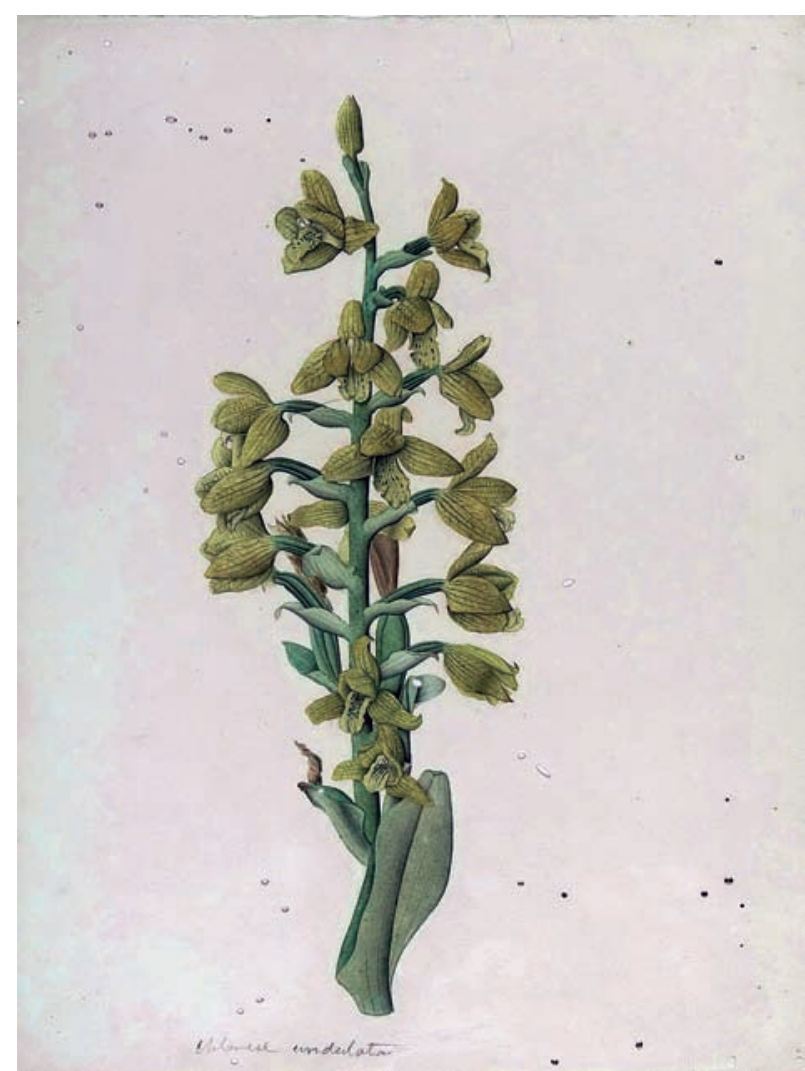

Figura 8. Chloraea undulata. Acuarela de C. undulata pintada por H. del Garnier (reproducida con permiso del Museo Antonio Raimondi).

cobertura natural de lomas ha retrocedido y se ha fragmentado de manera progresiva.

Un ejemplo notable de este proceso, es lo sucedido con el complejo de Lomas de Atocongo. Dicho sistema, que incluía antiguamente en una sola unidad vegetacional a las lomas del Lúcumo, Zorritos, Villa Maria y Retamal; hoy se encuentra dividida en 5 lomas que presentan, en sus zonas más bajas, la intrusión urbana de 4 de los distritos más poblados de Lima, el cementerio más grande del Perú y una gran concesión de minería no metálica.

Las faldas del cerro Amancaes en el distrito de Rímac, exhibían una cubierta estacional de "amancaes" que inspiró una de las fiestas costumbristas más populares de la Lima virreinal y republicana (Collantes \& Leon 1997). Hoy en día, calles y avenidas han reemplazado a las plantas de lomas. Ismene amancaes, la flor que dio el nombre al cerro, no ha vuelto ser encontrada en esta localidad. Los cerros seńalados por Raimondi, Weberbauer y Maish (Trujillo 2013) como localidades tipo donde C. undulata fue colectada y descrita (Cerro Amancaes, Cerro El Agustino y Cerro San Jerónimo forman actualmente parte del eje urbano central de la ciudad de Lima (Collantes \& Leon 1997). A consecuencia de dichas amenazas, la "orquídea de Lima” y otras especies de flores propias de las lomas de la costa central han visto mermadas sus poblaciones llegando, en ciertos casos, a registrarse como muy escasas o desaparecidas (Roque y León 2006).

Chloraea undulata está incluida en la lista de la Convención de Especies Amenazadas por el Comercio- CITES, siendo objeto de un tratamiento especial al igual que los otras tres orquídeas registradas para las formaciones de lomas: Aa weddelliana (Rchb.f.) Schltr., Pelexia matucanensis (Kraenzl.) Schltr. y Malaxis andicola (Ridl.) Kuntze (Trujillo 2013).

La Comunidad Campesina de Asia ha promovido la puesta en valor de este importante ecosistema, estableciendo circuitos ecoturísticos, controlando la frecuencia e intensidad de visitantes, estableciendo guardaparques comunales, modificando rutas de pastoreo y evitando la sobre extracción de recursos. La implementación de dichas acciones ha conducido a la aparición de especies vegetales que no se veían desde hace muchos años, entre ellas la "orquídea de Lima".

Contar con esta especie emblemática ha alentado la conservación de las lomas costeras a nivel local y regional, creando un sentido de identidad relacionado a un recurso natural que necesita ser protegido, y revalorando en simultáneo, el conocimiento ancestral heredado de tiempos prehispánicos, respecto a la conservación de las lomas.

Luego de dos ańos de trabajo dedicado, la presencia de la "orquídea de Lima" en el territorio de la Comunidad Campesina de Asia, ha compensado el esfuerzo de los pobladores locales. Este ejemplo espontaneo de gestión participativa, puede adoptarse como instrumento de política pública y ser replicado con éxito en otras lomas vecinas.

\section{Agradecimientos}

A la Comunidad Campesina de Asia, al Dr. Michael Dillon su constante apoyo y acertados comentarios, a Franco Pupulin por sus sugerencias y datos complementarios sobre las orquídeas de Ruiz y Pavón, a la Dra. Christine Giannoni, Bibliotecaria del Field Museum, a Hipólito Castillo y Ricardo Fernández, curador del herbario del Museo de Historia Natural (USM), por facilitarme el examen de las especies de Chloraea presentes en la colección Raimondi.

\section{Literatura citada}

Brako L. \& J.L. Zarucchi 1993. Catalogue of the Flowering Plants and Gymnosperms of Peru. Monogr. Syst. Bot. Missouri Bot. Gard. 45: 1-1286.

Cisternas M., G. Salazar, G. Verdugo, et al. 2012. Phylogenetic Analysis of Chloraeinae (Orchidaceae) Based on Plastid and Nuclear DNA Secuences. Botanical Journal of the Linnean Society. 168: 258-277.

Collantes B. \& M. Leon 1997. Chloraea undulata. The Orchid of Lima. The Lima Times 22:1.

Correa M. 1969. "Chloraea” Género Sudamericano de Orchidaceae. Darwiniana $15: 374-500$.

Colunga M. F. 1878. Lecciones de Botánica. Imprenta del Estado. Lima

Dillon, M. O. 2014. (en línea). LOMAFLOR Searchable Database $<$ http://emuweb.fieldmuseum.org/botany/search_lomas. php>. Acceso 01/11/14.

Dillon, M.O., S. Leiva, G.M. Zapata, et al. 2012. Floristic Checklist of the Peruvian Lomas Formations - Catálogo Florístico de las Lomas Peruanas. Arnaldoa 18(1): 7-32.

Garay L.A. \& G.A. Romero-González. 1998. Schedulae Orchidum. Harvard Papers in Botany 3(1): 53-62.

Ley $N^{\circ}$. 28611. 2005. Ley General del Ambiente. Normas Legales, El Peruano. Lima 15 de octubre de 2005. 302291-302310.

Lleellish M., J. Odar \& H. Trinidad. 2015. Guía de Flora de las Lomas de Lima. Servicio Nacional Forestal y de Fauna Silvestre. Lima.168 pp. doi: 10.13140/RG.2.1.4431.0564

Pupulin F. 2012. The Orchidaceae of Ruiz \& Pavón’s "Flora Peruviana et Chilensis". A Taxonomic Study. I. Anales Jard. Bot. Madrid 69(1): 21-79. doi:10.3989/ajbm.2295. 
Raimondi A. 2010. Flora perpetua: Arte y Ciencia Botánica de Antonio Raimondi. Luis Felipe Villacorta ed. Asociación educacional Antonio Raimondi. Lima

RM N 0274-MINAGRI. 2013. Reconocen y disponen la Inscripción en la Lista de Ecosistemas Fragiles del Ministerio de Agricultura, de la Loma de Lúcumo, ubicada en el Departamento de Lima. Lima 01 de agosto del 2013. El Peruano Normas legales: 500612-500613.

Roque J. \& B. León. 2006. Orchidaceae Endémicas del Perú. Revista Peruana de Biología. Número especial 2006; 13(2): 759 878. doi:10.15381/rpb.v13i2.1953

Sagastegui A. \& M. O. Dillon. 1991. Inventario Preliminar de la flora del Bosque Montesco. Arnaldoa 1 (1): 35-52.

Shweinfurth C. 1958. Orchids of Peru. Fieldiana, Bot. 30: 1 -531

Schweinfurth C. 1970. First supplement to the Orchids of Peru. Fieldiana, Bot. 33: 1-85.
The Plant List 2010. <http://www.theplantlist.org/> Acceso $15 / 06 / 2015$.

Trinidad H., E. Huaman, A. Delgado, et al. 2012. Flora Vascular de las Lomas de Villa Maria y Amancaes. Revista Peruana de Biología. 19 (2): 149-158. doi:10.15381/rpb.v19i2.834

Trujillo D. \& A. Delgado. 2011. Aa from Lomas Formation. A New Orchidaceae Record from Desert Coast of Peru. Lankesteriana 11 (1): 33-38. 2011. doi:10.15517/lank.v11i1.18313

Trujillo D. 2013. Diversidad de Orquídeas de las diferentes Formaciones Vegetales de los Andes peruanos. Lankesteriana 13 (1-2): 103-111. doi:10.15517/lank.v0i0.11546. 